States and internationally, to generate a global 'climate-information system.' The fact that operational satellites must meet needs other than those of scientists - a cornerstone of the GMES approach - is no obstacle to research, he says. Researchers just need to get involved to make sure their needs are taken into account.

The data that such information systems could make available will have implications both for how well scientists can run environmental models and for what those models can do. Operational systems launched in the late-2010s and onwards are likely to generate order-of-magnitude improvements in both temporal and spatial resolution. Today, a typical weather model might have 30-50-kilometre horizontal resolution. Climate research models are even coarser - often 100-200 km. But by 2025 , improvements in both data and computing will mean that weather will be modelled at $1-\mathrm{km}$ resolution, and climate models at 5-10 km, predicts Anthes.

Anthes has high hopes for the impacts such improvements could allow. "We have seen beyond a doubt in weather prediction that as the resolution increases, something almost magical starts to happen in the models, even without an increase in the observations," says Anthes. "As we go for example from a $30-\mathrm{km}$ resolution model to a $5-\mathrm{km}$ model, hurricane prediction, precipitation patterns and so forth become far more realistic."

More data obviously demand more computing power to make sense of them - and computing power is already the overriding limitation on how realistic models are, say many researchers. "It's a major issue," says Trenberth. To get good results you need to run the same model again and again with slightly different inputs, which eats up computing power. And every twofold increase in resolution requires a tenfold increase in the

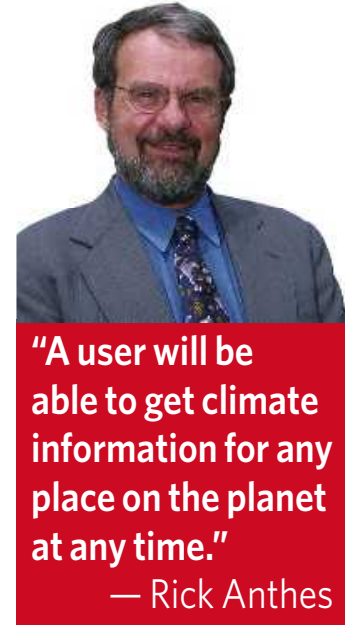

teraflops required. "Climate $\frac{\alpha}{<}$ prediction is probably the most $\stackrel{S}{\supset}$ computationally challenging problem in science," says Tim Palmer, a scientist at the European Centre for Medium-Range Weather Forecasts (ECMWF) in Reading, UK.

But Palmer is optimistic that the bottleneck will soon be alleviated. He points to the arrival next year of the first petaflop computers, running at peak speeds of up to 3,000 teraflops (see Nature 448, 6-7; 2007). By way of comparison, the ECMWF's fastest machines today run at less than a hundredth of that. Palmer predicts that by 2010, 10-petaflop machines will allow climate scientists who can get hold of them to run century-long simulations of the climate at $10-\mathrm{km}$ resolution. That could be $1 \mathrm{~km}$ within a decade after that. Accurate modelling of cloud processes at the $1-\mathrm{km}$ level, a key component currently missing from

\title{
Observing the ocean from within
}

A s names go, it's cleverer than most. At the mundane level, Argo stands for Array for Real-time Geostrophic Oceanography. But the name comes with not just one but two classical allusions attached. There's the Argo, the ship in which Jason sailed to find the golden fleece, a nod to the fact that the buoys that make up Argo were conceived as a counterpart to a satellite called Jason (see 'The Jason project'), which measures the surface topography of the oceans. And then there's Argos the giant, who was blessed with 100 eyes to see in all directions, with only a handful asleep at any one time. The modern Argo puts even that spectacularly distributed sensory system to shame - it has 3,000 different sense organs spread all around the globe (see map).

The array's 3,000-odd autonomous floats, which look like upended torpedoes, are equipped with sensors for recording temperature and salinity in the upper 2,000 metres of the ocean. Each float sinks, drifts, bobs up and transmits data to satellites on a regular basis. At its current size the array provides more than 100,000 temperature and salinity profiles each year, regardless of the season or weather. This is 20 times greater than the comparable annual measurements by research vessels and merchant ships, which in the past have been the main data source for this type of oceanography. In the past five years, Argo has

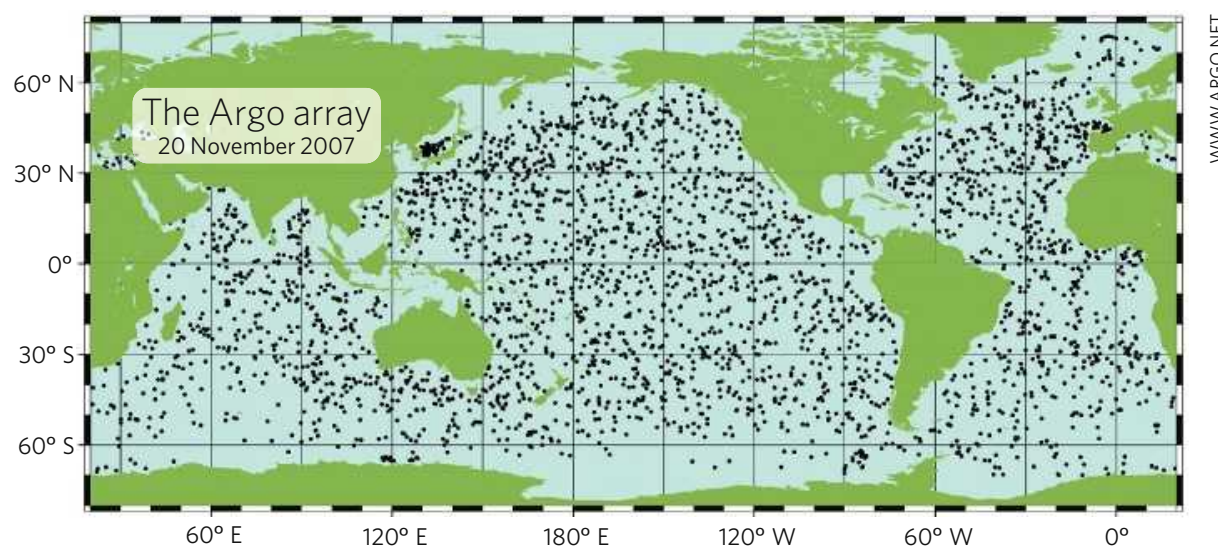

more than doubled the total database on some seas that ships steer clear of — such as the seas around Antarctica in winter.

Over the next years, the array's design will be reassessed with an eye to the need for additional floats and sensors. Already, 60 of the floats carry oxygen sensors - will that number be increased? What about sensors for particulate carbon, or for chlorophyll? Such extras are attractive, but always come at the cost of increased demand on the floats' batteries and thus a shorter lifetime.

Another idea is to design floats that can dive to greater depths. And special floats for polar latitudes - tethered to ice floes or equipped with acoustic sensors that tell the instrument where it can safely surface - are already being tested in seasonally ice-covered regions.

\section{Plugging the gaps}

Predicting and closing the gaps that will constantly appear in the array will be a major logistical challenge. The types of floats currently in use have a lifetime of 4 years, meaning that around 800 instruments need to be replaced each year. And replacements need to maintain the system's global coverage. That means dropping floats out of low-flying aircraft or chartering ships to optimal sites for replenishment.

The array's annual costs - around US $\$ 24$ million - are being shared by more than 30 nations. 
global climate models, could vastly improve predictions of regional climate change. Trenberth is one of a group of researchers planning to propose the creation of one or more international multi-petaflop computing facilities for climate prediction, with a ball-park cost of $\$ 1$ billion over 5 years. The idea will be presented at the international climate negotiations opening in Bali, Indonesia, this week, with a formal proposal to be published next year in Bulletin of the American Meteorological Society.

\section{Integrate and accumulate}

The data expected will not just be more precise - they will also be more wide-ranging, providing new impetus to models that seek to treat the Earth system as an integrated whole. Until recently, Earth observation has been less than the sum of its oceanic, terrestrial and atmospheric parts, according to Stephen Briggs, head of science, applications and future technologies at ESA. "Integrating the components is something we are really bad at," says

Only Germany, however, has so far made a firm long-term funding commitment. In the United States, which contributes $50 \%$ of the overall programme costs, funding currently comes through the National Oceanographic and Atmospheric Administration. But it is not yet clear which agency will be responsible for maintaining the array once Argo becomes a routinely operational system. Proof of definite advantages - for fisheries, merchant shipping, oil-spill management or naval purposes - could help secure long-term funding from more governments, says John Gould, a consultant and former director of the project. "But it's still rather early days to evaluate the benefits."

\section{Raft of achievements}

The first scientific achievements using data from Argo include the detection of accelerated circulation in the subtropical South Pacific ${ }^{1}$, the tracking of the deep water that forms in the Labrador Sea ${ }^{2}$ and the observation of local sea-temperature changes during hurricanes. But there have also been setbacks. A surprising suggestion that the oceans' heat content was diminishing, rather than increasing as expected $^{3}$, turned out to be an artefact caused by a software glitch ${ }^{4}$.

Gould says that this underlines the need for experienced scientists - who know both the oceans and the technology - to be involved in data management. "We're still learning," he says.

Argo data are already incorporated into models for seasonal weather prediction. To initialize such forecasts, scientists 'tell' their
Briggs. "This is where we are going to see the major advances."

Incorporating more geophysical observations made from multiple instruments obviously makes models more complex. To integrate such disparate data sets, which differ not just in their spatial and temporal resolution but also in their error profile, modellers are borrowing the 'data assimilation' techniques used by weather forecasters. A model producing a weather forecast will start off with reasonable best estimates of initial global conditions informed by the data to hand. As more come in - as low-Earth-orbit

\section{The Jason project}

Jason is a joint French/US satellite

designed to monitor the oceans' topography.

The project follows on from TOPEX/

Poseidon, an earlier collaboration between

the French space agency CNES and NASA,

in which a French and an American radar

altimeter shared the same antenna. Jason

is an operational continuation of that earlier research effort. Its successor Jason-2

will move yet farther down the road to

operational use, bringing in EUMETSAT, the

US weather-satellite operation.

Ocean altimetry provides

data on sea level and on

ocean currents, which

deform the surface.

There is a significant

synergy between

Jason's data on

the surface of the

ocean and those

from the Argo

float array on

the ocean's inner

structure.

Oliver Morton

models about the here and

now and then cast them off into

the future. Precise knowledge of the initial state of the ocean - which has a longer 'memory' than the atmosphere - could greatly improve the accuracy of longer-term weather and climate prediction. At the UK Met Office's Hadley Centre in Exeter, Argo data have been used to initialize the centre's new decadal temperatureforecasting system, which combines observations and models on longer timescales ${ }^{5}$.

"The data are absolutely invaluable," says Matt Huddleston, a climate scientist at the Hadley Centre. "We now use Argo data continuously for forecasting everything from European winters to tropical storms and El Niño events." The success of the Met Office in correctly predicting some very cold and wet conditions during the 2005-2006 European satellites pass over new places, for example the model's evolution is reiteratively compared with reality. So sparse and infrequent sources of data can still play a role.

Counter-intuitively, more data sources can also often simplify modelling, as they can help to better define other variables, adds Trenberth. For example, raw measurements from a buoy might be misleading if it were in an eddy of the warm Gulf Stream rather than somewhere more representative of the Atlantic as a whole. A system that could use other data to know that the buoy was in the Gulf Stream would not be misled so easily, and the model would be made more realistic. "As one can resolve features better," he predicts, "one can utilize data better." The resolution and the data can be provided, if the institutions allow; it will then be up to Trenberth and his colleagues to make good on that forecast.

Declan Butler is a senior reporter for Nature, based in France.

See Editorial, page 761.

winter seems to have been made possible in part by Argo data, which revealed an abnormal subsurface temperature pattern in the North Atlantic. But statistical proof of such benefits will be available only when the Argo network has been maintained for much longer.

Perhaps Argo's most valuable contribution will be in facilitating studies of year-to-year and decade-to-decade variability in the oceans. At present, it is hard to distinguish climate-change-driven shifts in ocean circulation from natural fluctuations. Argo will provide the continuous time-series needed to solve such puzzles. It should also help settle the big question of the extent to which the oceans participate in climate variability.

"We keep learning that it is dangerous to infer changes in the oceans from only a few years of measurements," says Brian King, a physical oceanographer at the Southampton Oceanography Centre, UK. "But if anthropogenic forcing does leave a mark on the ocean, Argo should definitely help us find out."

\section{Quirin Schiermeier}

Roemmich, D. et al. J. Phys. Oceanogr. 37, 162-173 (2007)

2. Fischer, J. \& Schott, F. J. J. Phys. Oceanogr. 32, 573-584 (2002).

3. Lyman, J. M., Willis, J. K. \& Johnson, G. C. Geophys. Res. Lett. 33, L18604 (2006).

4. Nature $447,8-9$ (2007)

5. Smith, D. et al. Science 317, 796-799 (2007). 